Hadron Structure '15

International Journal of Modern Physics: Conference Series

Vol. 39 (2015) 1560098 (7 pages)

(C) The Author(s)

DOI: $10.1142 /$ S2010194515600988

\title{
Compressed baryonic matter at FAIR: JINR participation
}

\author{
P. Kurilkin* $\ddagger, \S$, V. Ladygin*, A. Malakhov* and P. Senger ${ }^{\dagger}$ \\ *JINR, Joliot-Curie 6, 141980, Dubna, Russia \\ ${ }^{\dagger}$ GSI, Planckstraße 1, 64291 Darmstadt, Germany \\ ${ }^{\ddagger} F S B I$ "SSC RF ITEP" of NRC "Kurchatov Institute" \\ 117218, Moscow, Russia \\ §pkurilkin@jinr.ru
}

Published 26 November 2015

\begin{abstract}
The scientific mission of the Compressed Baryonic Matter(CBM) experiment is the study of the nuclear matter properties at the high baryon densities in heavy ion collisions at the Facility of Antiproton and Ion Research (FAIR) in Darmstadt.

We present the results on JINR participation in the CBM experiment. JINR teams are responsible on the design, the coordination of superconducting(SC) magnet manufacture, its testing and installation in CBM cave. Together with Silicon Tracker System it will provide the momentum resolution better $1 \%$ for different configuration of CBM setup. The characteristics and technical aspects of the magnet are discussed. JINR plays also a significant role in the manufacture of two straw tracker station for the muon detection system. JINR team takes part in the development of new method for simulation, processing and analysis experimental data for different basic detectors of CBM.
\end{abstract}

Keywords: Heavy ion collisions; baryon matter.

PACS Numbers: 25.75.Dw, 84.71.Ba

\section{Introduction}

The investigation of nuclear matter at high temperatures and/or at high baryon densities is one of the most challenging fields of modern physics. The significant experimental and theoretical efforts in the world are devoted to the exploration of the phase diagram of nuclear matter. The QCD phase diagram demonstrate a rich structure at finite values of baryon chemical potentials, such as the critical point, the predicted first order phase transition between hadronic and partonic matter, and the chiral phase transition. The experimental discovery of these landmarks of the QCD phase diagram would be a major breakthrough in our understanding of nuclear matter properties.

This is an Open Access article published by World Scientific Publishing Company. It is distributed under the terms of the Creative Commons Attribution 3.0 (CC-BY) License. Further distribution of this work is permitted, provided the original work is properly cited. 
Pioneering heavy-ion experiments has been curried out at AGS in Brookhaven ${ }^{1}$ and at low CERN-SPS beam energies ${ }^{2}$ in order to explore the QCD phase diagram at large baryon-chemical potentials. The STAR collaboration at RHIC scanned the beam energies in order to search for QCD critical endpoint. ${ }^{3}$ For the same reason, the NA61/SHINE experiment at CERN-SPS continues to search the first-order phase transition using light and medium size beams. ${ }^{4}$ Neither NA61 at SPS nor STAR at RHIC will be able to run at beam energies of $10 \mathrm{AGeV}$ and less. At the Joint Institute for Nuclear Research in Dubna, a fixed target experiment is under preparation at the Nuclotron in order to investigate the heavy-ion collisions at gold-beam energies up to $4 \mathrm{AGeV}$. Moreover, the collider facility NICA with multipurpose detector(MPD) is planed at JINR. ${ }^{5}$ However, due to luminosity or detector limitations these experiments are constrained to the investigation of abundantly produced particles.

In contrast, the Compressed Baryonic Matter (CBM) experiment ${ }^{6}$ at the future FAIR accelerator in Darmstadt is designed to run at extremely high interaction rates (up to $10 \mathrm{MHz}$ ). In particular, the CBM research program aims at the exploration of the structure of high density matter including the question of deconfinement and chiral phase transitions. The theoretical description of physics at high net baryon densities within the fundamental theory of strong interaction, Quantum Chromodynamics (QCD), is still strongly evolving and the scientific progress in "strong" nonperturbative QCD is driven by new experimental data. The CBM experiment will enter a new era with diagnostic probes never measured before in the FAIR energy range, and thus has a unique research potential.

\section{CBM Experimental Setup}

The CBM detector is designed as a multi-purpose device which will be able to measure hadrons, electrons and muons in heavy-ion collisions over the full SIS100/SIS300 beam energy range. The geometrical acceptance of the CBM detector systems is full $2 \pi$ for azimuth $(\phi)$ and from $2.5^{\circ}$ to $25^{\circ}$ for polar $(\Theta)$ angles. Muon and electron pairs created during particle decays will be measured during separate runs. In electron configuration following detectors will be used: Micro-vertex Detector (MVD), Silicon Tracking System (STS), both placed in gap of superconducting (SC) magnet, then Ring Imaging Cherenkov Detector(RICH), Transition Radiation Detectors(TRD), Resistive Plate Chambers for time-of-ight measurements, Electromagnetic Calorimeter(ECAL) and Projectile Spectator Detector(PSD). In muon configuration the RICH detector will be replaced by the Muon Detection System( $\mathrm{MuCh})$ and ECAL will be removed. The detailed description of CBM detector can be found in Ref. 7 .

\section{JINR Participation in CBM}

The physicists from JINR are participating in the several sub-projects of CBM experiment.The design and production of SC dipole magnet, the development and 
production of a straw tube tracker and the study of multiparticle dynamics in heavy-ion collisions at CBM are the main directions.

\subsection{SC dipole magnet}

The physicists from JINR are responsible for the design, control of the manufacture, maintenance and commissioning of the superconducting dipole magnet. ${ }^{8,9}$ A perspective view of the CBM magnet with the support is shown in Fig. 1. It is H-type with a warm iron yoke/pole and cylindrical superconducting coils in two separate cryostats. The CBM magnet has a large gap $(1.4 \times 2.5 \mathrm{~m})$ in order to accommodate The target, the MVD and STS. The magnet coils will provide a magnetic field with total bending power $1 \mathrm{Tm}$ over a length of $1 \mathrm{~m}$ from the target. Design calculation for the CBM superconducting dipole magnet were performed using the code ANSYS for the structural analysis for the coil case, the coil vessel and the support links, while the calculation of the electromagnetic forces exerted on the coil was made using the code TOSCA.

The magnet will store about 5.15 MJ at its operating current of $686 \mathrm{~A}$. The quench simulations ${ }^{10,11}$ are necessary to design the SC coil and quench protection system of CBM dipole magnet. Figure 2 presents the results of the quench simulation in the case when all storage energy of the CBM magnet dissipated in the coil. The maximum quench voltage reaches $1240 \mathrm{~V}$ and the maximum hot-spot temperature equals $175 \mathrm{~K}$. It is planned to use the quench protection system of CBM magnet based on the the extraction of the storage energy in an external dump resistor to minimize the coil peak temperature and to avoid vaporization of helium.

\subsection{Design of straw tube tracker}

Soft muons from vector meson decays will be measured with an active hadron absorber system (see Fig. 3) consisting of 6 iron slabs of different thickness and muon

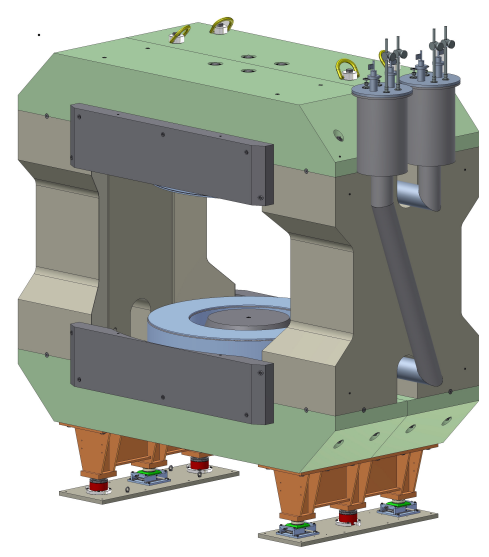

Fig. 1. Perspective view of CBM dipole magnet with the support. 

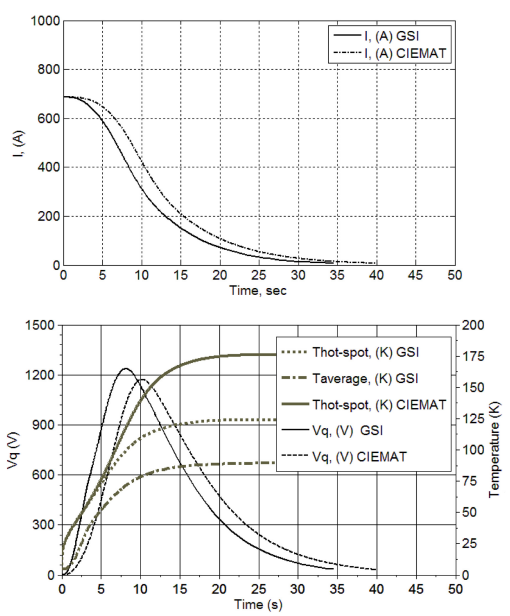

Fig. 2. Results of 3D quench simulation for CBM magnet.

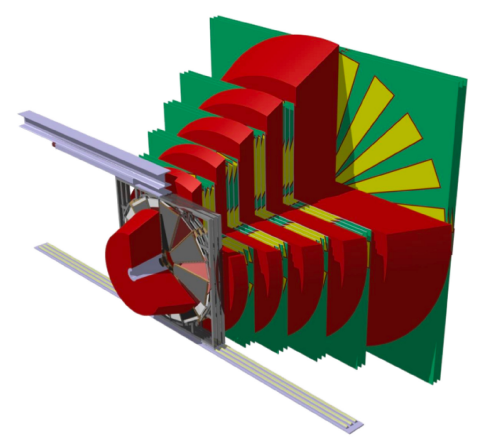

Fig. 3. Perspective view of $\mathrm{MuCh}$ detector.

tracking chambers behind each iron absorber. The large-size triple GEM detectors will be used for the first few station of CBM-MuCh system because of high particle density (up to $500 \mathrm{kHz} / \mathrm{cm} 2$ ). Further downstream, where the hit density is reduced, straw-tube detectors will be used. ${ }^{12}$ Schematic view of the MUCH station with three straw chambers is presented in Fig. 4. Several straw tube prototypes and full size prototype of straw detectors have been designed, produced and tested by JINR physics. They also started the development of new application specific integrated circuit (ASIC) for Front-End Electronics(FEE).

\subsection{Multiparticle dynamics studies in heavy-ion collisions at CBM}

The goal of the multi-particles dynamics studies at CBM at SIS100 and SIS300 energy ranges is to perform the simulation and feasibility studies for different 


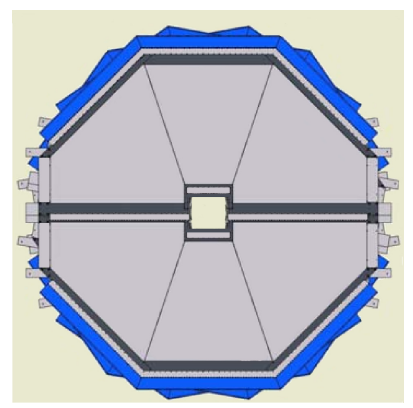

Fig. 4. Schematic view of the MUCH station with three straw chambers.

observables with hadrons and nuclear fragments to sensitive the details of the nuclear matter modification in heavy-ion collisions. The several observables where investigated during several years: the high transverse momenta spectra, di-hadron correlations, nuclear fragments and hyper-nuclei production. The suppression of particle production at high transverse momenta in central nucleus-nucleus reactions has been discovered at RHIC in central nucleus-nucleus reactions relative to peripheral ones as well to $p+$ nucleus and $p+p$ collisions, ${ }^{13,14}$ that is generally interpreted as a sign of parton energy loss in hot and dense strongly interacting matter. The energy dependence of these effects was investigated via a systematic study of $\mathrm{Pb}+\mathrm{Pb}$ reactions at top SPS energy, $158 \mathrm{AGeV}$ with the NA49 detector at CERN. ${ }^{15}$ The study of the particle production at high transverse momenta $p_{T}$ in central heavy-ion collisions at CBM can also give the information on the partonic phase of strongly interacting matter. The simulation of high momentum transverse spectra of positively and negatively charged pions for the central $A u+A u$ collisions at 3.5-25 A GeV at CBM setup has been performed. ${ }^{16}$ It has been shown that RICH information can be used for high momentum pions selection at SIS100/SIS300 energies. $^{17}$

Two particle correlation observables provide a unique access to the spatial extension and dynamics of the source of particles emitted during heavy-ion collisions. Measurements of the azimuthal correlation $C_{2}(\Delta \phi)$ can provide the information of the properties modification of mini-jets originating from hard scattering partons. Recent RHIC results ${ }^{19}$ on high $p_{T}$ di-hadron correlations confirmed that this approach can be used to study the properties of the nuclear medium. At CERN SPS energies the so called hole-jet transition in di-hadron correlations has been observed. ${ }^{20}$ Such investigations can be performed at CBM at SIS-100 energies. ${ }^{21}$ Figure 5 presents the azimuthal correlation $C_{2}(\Delta \phi)$ for the pions obtained for $10 \mathrm{k}$ central $A u+A u$ UrQMD 2.3 events at $10 \mathrm{AGeV}$. The $C_{2}(\Delta \phi)$ distribution is close to the unity. One can expect the away-side enhancement for systems with smaller size (for instance, $S i+S i^{20}$ ).

Systematic studies of the light nuclear fragments and hyper-nuclei production in heavy-ion collision could distinguish different approaches in the description of 


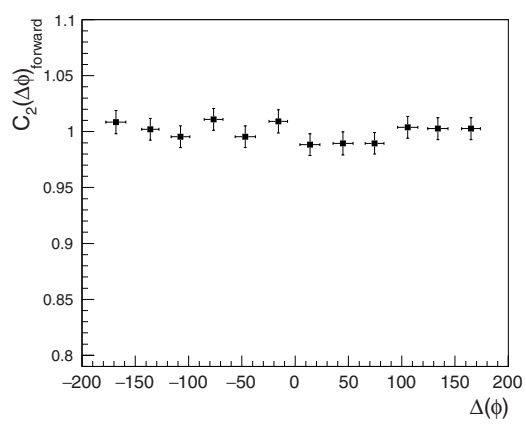

Fig. 5. Azimuthal correlation $C_{2}$ for pions for $A u+A u$ UrQMD2.3 central events at $10 \mathrm{AGeV}$ as a function of $\Delta \phi$.

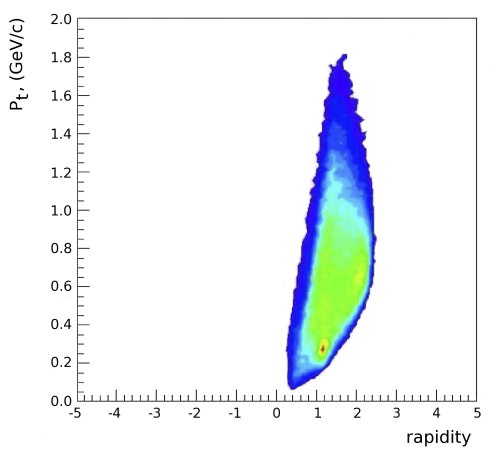

Fig. 6. $y p_{T}$ distribution for deuterons from minimumbias $A u+A u$ collisions at $4 \mathrm{~A} \mathrm{GeV}$.

these processes. ${ }^{23}$ The CBM setup at SIS100 allows to obtain data on the light nuclei production in the mid- and forward rapidity regions. ${ }^{22}$ Figure 6 presents the transverse momentum-rapidity distribution for deuterons from minimum-bias $\mathrm{Au}+\mathrm{Au}$ collisions at $4 \mathrm{~A} \mathrm{GeV}$. Secondary deuterons were selected using the reconstructed momentum and time-of-flight information from STS and TOF of CBM setup, respectively.

\section{Conclusion}

Compressed Baryonic Matter experiment is devoted to the third generation of the heavy ions collision experiments. Its research program aims at the exploration of the structure of high density matter including. For these purposes the advanced experimental setup will be build for high counting rate conditions expected at FAIR.

JINR contribution in CBM project is significant and can be done in the development of the physics program, data and simulation analysis algorithms, SC dipole 
magnet and straw tube tracker of the CBM setup. These subsystems are the parts of first stage of the CBM setup at SIS100.

\section{Acknowledgments}

The work has been supported in part by the BMBF-JINR and FRCC grants.

\section{References}

1. C. Pinkerburg et al., Phys. Rev. Lett. 83, 1295 (1999).

2. C. Alt et al., Phys. Rev. C 77, 024903 (2008).

3. A. Schmah, arXiv:1202.2389v1 (2012).

4. A. Laszlo et al., (The NA61 Coll.), NA61/SHINE at the CERN SPS, Pos(CPOD07)054, (2007).

5. http://nica.jinr.ru

6. B. Friman et al., Compressed Baryonic Matter in Laboratory Experiments, Lecture Notes in Physics 814 1st Edition., 960 (2011).

7. CBM collab. (T. Balog), Journal of Physics: Conference Series 503, 012019 (2014).

8. CBM Collab. (P.Akishin et al.), Technical Design Report for the CBM Superconducting Dipole Magnet, Darmstadt 2014.

9. A. Bychkov et al., The SC Dipole Magnet for the CBM Experiment at FAIR, CBM Progress Report 2014, Darmstadt 2015, p. 12.

10. P. Kurilkin et al., 3D CIEMAT Code Optimization for the CBM Magnet Quench Calculation, CBM Progress Report 2014, Darmstadt 2015, p. 13.

11. P. Kurilkin et al., Quench Calculation for the CBM Dipole Magnet, CBM Progress Report 2013, Darmstadt 2014, p. 9.

12. V. Peshekhonov et al., Phys. Part. Nucl. Lett. 9, 172 (2012).

13. PHENIX Collab. (S. S. Adler et al.), Phys. Rev. C 74, 024904 (2006).

14. STAR Collab. (L. Ruan et al.), J. Phys. G 31, 1029 (2005).

15. NA49 Collab. (C. Alt et al.), Phys. Rev. C 77, 034906 (2008).

16. V. Ladygin et al., Study of High $p_{T}$ Pion Emission in Central $\mathrm{Au}+\mathrm{Au}$ Collisions at CBM CBM Progress Report 2010, Darmstadt 2011, p. 76.

17. V. Ladygin et al., Identification of High-Momentum Pions with RICH CBM Progress Report 2011, Darmstadt 2012, p. 77.

18. The PHENIX Collab. (A. Adare et al.), Phys. Rev. C 78, 014901 (2008).

19. STAR Collab. (H. Agakishiev et al.), arXiv:1010.0690 [nucl-ex].

20. NA49 Collab. (M. Szuba et al.), arXiv:0809.5210v1 [nucl-ex].

21. V.Ladygin et al., Study of $C_{2}(\Delta \phi)$ Azimuthal Correlation in Central Au $+A u$ Collisions at CBM, CBM Progress Report 2010, Darmstadt 2011, p. 77.

22. V. Ladygin et al., Nuclear Fragments with CBM at SIS100, CBM Progress Report 2014, Darmstadt 2015, p. 122.

23. J. Steinheimer et al., Phys. Rev. B 714, 85 (2012). 